\title{
Electromagnetic physical modeling of a gallium nitride distributed transferred electron based planar waveguide structure THz oscillator
}

\author{
Christophe Dalle
}

Institut d'Electronique, de Microélectronique et de Nanotechnologies, France
*corresponding author, E-mail: Christophe. dalle@univ-lille.fr

\begin{abstract}
The potential of a planar waveguide structure terahertz oscillator based on a gallium nitride distributed transferred electron device is theoretically investigated. The circuit numerical physical modeling relies on a two-dimensional time-domain electromagnetism/transport simulator. It is based on the coupled solution of the Maxwell and energymomentum macroscopic transport equations. The study is focused on the analysis, from both the space-time electromagnetic and electron transport quantities, of the complex CW operation of an oscillator, designed and DC biased, to optimally operate at one terahertz. The analysis is performed following a full electromagnetic approach in the time and frequency domain, at the local scale, for the description of the physical phenomena, as well as at the functional scale in order to obtain the quantities interesting the oscillator designer and user.
\end{abstract}

\section{Introduc tion}

The development, on a large scale, of $\mathrm{THz}$ applications remains still today dependent on the availability of solid-state $\mathrm{THz}$ power sources, that are powerful enough, low cost, monolithically integrated and safely operating at ambient temperature. A lot of solution are presently under study as well in term of RF operation (direct generation, frequency multiplication, optical mixing...) as in term of semiconductor structures. Among the possible electronic (transistor, varactor ...) and optical (laser, quantum cascade laser ...) solutions is the transferred electron device (TED). Indeed, a great family of device can be derived from the concept of TED as well as in term of semiconductor structures (homo or heterojunction vertical, FET-like, Bipolar-like ...) as in term of $\mathrm{RF}$ operating modes (fundamental and harmonic generation, single or multitransit...). Indium phosphide (InP) and gallium arsenide (GaAs) TEDs have led to the realization of RF power sources up to millimeter wavelengths [1]. At higher frequencies and on the basis on the potential of the electron transport properties, gallium nitride $(\mathrm{GaN})$ TEDs are expected to operate at THz ones. Vertical lumped GaN TEDs have already been the purpose of both theoretical and experimental research works [2-4]. Theoretical studies predict significant performance while experimental ones are still limited to the demonstration of the existence of the semiconductor material negative differential mobility [5-6]. Indeed, a lot of research works is still to be done to overcome both the technological constraints, resulting from the epitaxial layer structure, and the technical ones, resulting from the electronic (load circuit matching, connection) and thermal (thermal resistance) limitations. All the works previously devoted to TEDs have concerned lumped structures. Another solution is the distributed TED (DTED).

The research works carried out by the past on distributed active semiconductor devices for the realization of high frequency RF power sources have mainly concerned the distributed impact ionization and avalanche transit time (DIMPATT) diodes. Theoretical modeling of increasing accuracy have been proposed [7-9]. They were based on linear and non-linear, analytical and transmission line modeling. Two-dimensional (2D) time-domain numerical physical modeling, based on the coupled solution of the Maxwell and macroscopic drift-diffusion equations for the free carrier transport model, has been also developed [10]. All these models have allowed for investigating the potential of microwave DIMPATT. Experimental works have validated the device concept and RF operation [11]. However, confronted with the enhancement of the transistor performance, the DIMPATT measured performance were not convincing enough and the studies were abandoned. By contrast, at $\mathrm{THz}$ frequencies, the competition between lumped and distributed devices is still open.

To our knowledge, few theoretical research works have been performed on the physical analysis of the DTED RF operation [12]. They were limited to the DTED semiconductor structure and based on a simplified quasielectrostatic approach. They nevertheless confirm the potential of the device that is a RF operation in accordance with the expected one. However, a realistic DTED oscillator modelling requires to account for the active device/passive load circuit interaction and this time be based on a full electromagnetic approach. Indeed, it can be expected from preliminary theoretical investigations based on physical modeling to limit the risk of disappointing and costly investments in an unproductive technological realization.

The DTED oscillator RF operation is complex. It is based on an EM wave propagating and interacting with free electrons moving under the accumulation layer and transit mode. The present study is focused on the analysis of the 
oscillator operation at THz frequencies. Because of the DTED highly non-linear RF behavior, depending on the local electron velocity/electric field interaction, the induced spacecharge effects and the electron relaxation effects resulting from the high frequency operation, an accurate as possible 2D time-domain numerical electromagnetic (EM) physical modeling has been developed [13]. It is described in the second section of the paper. The planar waveguide oscillator structure is described in the section 3 . In the fourth section, the DTED oscillator continuous wave $(\mathrm{CW})$ operation is considered from all angles. It is based on an exhaustive analysis of the space-time EM and electron transport quantities. It is carried out in the time domain but also, with the help of the Fourier analysis tools, in the frequency domain. Moreover, it is performed at local scale, for the description of the physical phenomena, as well as at a functional scale in order to obtain the quantities interesting the oscillator designer and user. Finally, we conclude on the feasibility and sound feature of the oscillator RF operation.

2. Simax_2

D simulator

\subsection{Electromagnetic modeling}

The $\mathrm{THz}$ oscillator modeling is a laboratory made time domain physical numerical software. It fundamentally consists of two main entities allowing the self-consistent solution of both the Maxwell and free electron transport equations in the time domain (Figure 1). The main determined quantities are the EM fields $\vec{E}$ and $\vec{H}$ in the whole considered $2 \mathrm{D}$ area and the free electron conduction density $\overrightarrow{J_{c}}$ limited to the semiconductor region.

\section{D time-domain Maxwell/Transport model}

\begin{tabular}{|l|l|l|}
\cline { 2 - 2 } Maxwell & \multicolumn{1}{|l|}{$\begin{array}{l}\text { Transport } \\
\text { Quasi-electrostatic } \\
\text { Electromagnetic }\end{array}$} \\
$\vec{E}=\vec{E}_{D C}+\vec{E}_{R F}$ & $\vec{E}, \vec{H}$ & $\begin{array}{l}\text { Semiconductor material } \\
\text { Macroscopic modeling: } \\
\text { Energy/momentum }\end{array}$ \\
$\vec{H}=\vec{H}_{D C}+\vec{H}_{R F}$ & $\vec{J}_{c}$ & \\
$\begin{array}{l}\text { Perfect metal } \\
\text { Dielectric interfaces } \\
\text { Absorbing boundary } \\
\text { conditions }\end{array}$ & \\
\end{tabular}

Figure 1: Simax_2D simulator main organization

The electromagnetic model is applied on a physical rectangular area in the $x$ and $y$ directions. It is split in quasielectrostatic and electrodynamic sub-entities (figure 1). Thus, the electric and magnetic fields in DC and RF components are expressed as:

$$
\begin{aligned}
& \vec{E}=\vec{E}_{D C}+\vec{E}_{R F} \\
& \vec{H}=\vec{H}_{D C}+\vec{H}_{R F}
\end{aligned}
$$

The electrodynamic model is used for the DTED RF operation simulation. It is based on the solution of the Maxwell/Faraday/Ampere equations:

$$
\begin{gathered}
\operatorname{rot} \vec{E}_{R F}=-\mu \frac{\partial \vec{H}_{R F}}{\partial t} \\
\operatorname{rot} \vec{H}_{R F}=\vec{J}_{c}+\epsilon \frac{\partial \vec{E}_{R F}}{\partial t}
\end{gathered}
$$

The quasi-electrostatic model is based on the Maxwell/Poisson/Ampere equations:

$$
\begin{aligned}
& \operatorname{rot} \vec{E}=0 \\
& \Delta V=-\frac{\rho}{\epsilon}
\end{aligned} \Rightarrow \vec{E}=-\operatorname{grad} V
$$

with $\rho=N_{D}-n$ in the semiconductor region

$$
\vec{J}_{t}=\vec{J}_{c}+\epsilon \frac{\partial \vec{E}}{\partial t}
$$

The quasi-electrostatic model has two main utilizations. It is firstly used to describe the DTED DC bias conditions. Thus, it allows determining the initial spatial distribution of the free electrons and the DC fields and current components $\vec{E}_{D C}, \vec{H}_{D C}, \vec{J}_{D C}$ as a function of the DC bias voltage $V_{D C}$ applied at the terminal boundaries of the semiconductor device. Secondly, when associated to the transport model, it constitutes a simplified but efficient time-domain semiconductor device model. Its use is justified when the EM wave propagation effects are negligible. Thus, it is presently used in a one-dimensional version in order to optimize, under CW pure sine RF operation, the epitaxial layer technological parameters in relation with the estimation of the best vertical TED potential RF performance [14].

\subsection{Free carrier transport model}

The DTED is a unipolar semiconductor device. Thus, the transport model is an electron physical model issued from the general Boltzmann transport equation [15]. It relies on the macroscopic approach leading to conservation equation systems determined by simplifying assumptions following the single electron gas approximation [16].

At $\mathrm{THz}$ frequencies, the electron inertia effects cannot be neglected anymore. Indeed, they tend to soften the involved physical phenomena to the detriment of the device potential. Consequently, the transport model is a non-stationary energymomentum model accounting for the electron relaxation effects [17]. It is based on the continuity (electron density), averaged total energy density and momentum density equations (here expressed in normalized form [18]): 


$$
\begin{aligned}
& \frac{\partial n}{\partial t}=\operatorname{div} \vec{J}_{n}+g-u \\
& \frac{\partial n \varepsilon_{n}}{\partial t}=\vec{J}_{n} \vec{E}+\operatorname{div}\left(n v_{n}\left(\varepsilon_{n}+k T_{n}\right)\right)-\frac{\left(\varepsilon_{n}-\varepsilon_{0}\right)}{\tau_{\varepsilon}} \\
& \text { with } \varepsilon_{n}=\frac{1}{2} m_{n}^{*} v_{n}^{2}+\frac{3}{2} k T_{n} \\
& \frac{\partial n m_{n}^{*} v_{n l}}{\partial t}=-n E_{l}-\operatorname{div}\left(n m_{n}^{*} v_{n l} \vec{v}_{n}\right)-\frac{\partial}{\partial l}\left(k T_{n}\right) \\
& -\frac{n m_{n}^{*} v_{n l}}{\tau_{m}}
\end{aligned}
$$

with $l \in\{x, y\}$

$$
\vec{J}_{n}=-n \vec{v}_{n}=\vec{J}_{c}
$$

\subsection{Semiconductor material electron transport parameters}

The energy-momentum modeling requires electron transport parameters which are the energy and momentum relaxation times, the electron effective mass as a function of the energy. They are classically obtained from bulk semiconductor material Monte-Carlo simulations performed under static steady-state operation through the energy/electric field characteristic $E_{S S}(\varepsilon)[19]$ :

$$
\begin{aligned}
\tau_{\varepsilon} & =\frac{\varepsilon-\varepsilon_{0}}{E_{S S}(\varepsilon) v_{s S}\left(E_{S S}(\varepsilon)\right)} \\
\tau_{m} & =\frac{m^{*}\left(E_{S S}(\varepsilon)\right) v_{s S}\left(E_{S S}(\varepsilon)\right)}{E_{S S}(\varepsilon)} \\
m^{*} & =m^{*}\left(E_{S S}(\varepsilon)\right)
\end{aligned}
$$

The potential accuracy of the macroscopic transport modeling not only depends on the model formulation but also fundamentally on the transport parameters. The GaN transport parameters used are detailed in the reference 12.

\subsection{Numerical Method}

The numerical method is the finite-difference/time-domain (FDTD) method. The spatial mesh is variable and the position of both the scalar and vector physical quantities is defined following the Yee's topology [20]. The Alternating Direction Implicit method (ADI) is systematically used [2122]. It leads to the numerical solution of simple tridiagonal matrix/vector equations [23]. For the transport equations, the spatial derivatives resulting from the diffusion terms of the equations are evaluated using the second order centered numerical approximation. That of the convection terms is based on the upwind method. The full implicit approximation is used for the time derivatives. The ADI method allows the use of a time increment value close to that imposed by the macroscopic transport models. It is determined by the dielectric relaxation time, calculation velocity, propagation velocity and carrier relaxations times. It must be here limited to $1 \times 10^{-16} \mathrm{~s}$. The space increments are mainly limited by the space gradients. They are as low as $2 \times 10^{-9} \mathrm{~m}$ in the carrier transport direction $y$ and typically $1 \times 10^{-6} \mathrm{~m}$ in the propagation direction $x$.

\section{DTED oscillator modeling problematic}

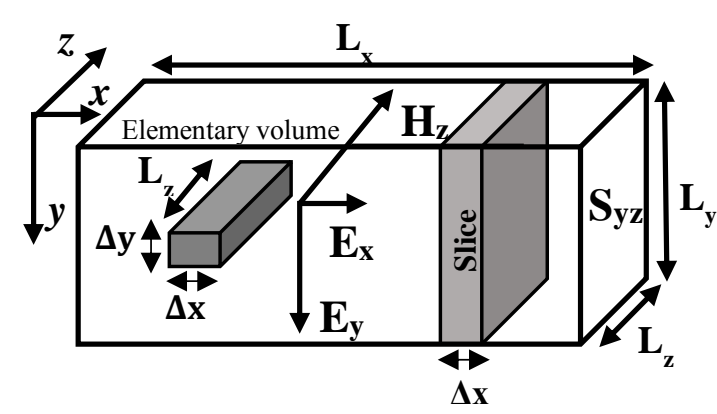

Figure 2: DTED oscillator geometrical parameters

The simplest DTED semiconductor structure is a microstrip propagation line. Accounting for its geometrical dimensions, its numerical modeling on its own in 3D is still out of capacity of our calculation means.

In $2 \mathrm{D}$ and from an electromagnetic point of view, the DTED structure is similar to that of a multi-layered parallel plate waveguide. From the electron transport point of view, the DTED is a classical flat doping profile $\mathrm{N}^{+} \mathrm{NN}^{+}$structure stretched following the $x$ propagation direction (figure 3 ). Note that the word "transverse" will be associated with phenomena occurring in the y direction or yz planes (figure 2). Those occurring in the $x$ direction will be associated with the terms "longitudinal", "lateral", "progressive" in the direction of the positive $\mathrm{x}$ values and "regressive" in the opposite direction.

The DTED RF operation is conceptually based on the interaction between an EM wave propagating in parallel to the $\mathrm{N}^{+} \mathrm{NN}^{+}$epitaxial layers in the $x$ direction and electrons moving perpendicularly in the $y$ direction. Under appropriate DC bias conditions, the EM wave amplification results from the transversal modulation of the electron motion under the action of the $y$ direction RF component of the electric field $E_{R F y}$. Thus, the EM wave propagates along the $\mathrm{N}$ active zone behaving, from a functional electrical point of view, as a transverse RF negative resistance like medium. This property fundamentally results from the semiconductor material presenting a negative differential mobility range in its electron velocity/electric field characteristic. The device is here structurally designed and DC biased to operate under the electron accumulation layer and single transit RF mode. The thermal problematic is not detailed in this paper and a constant junction operating temperature of $500 \mathrm{~K}$ is simply considered. 


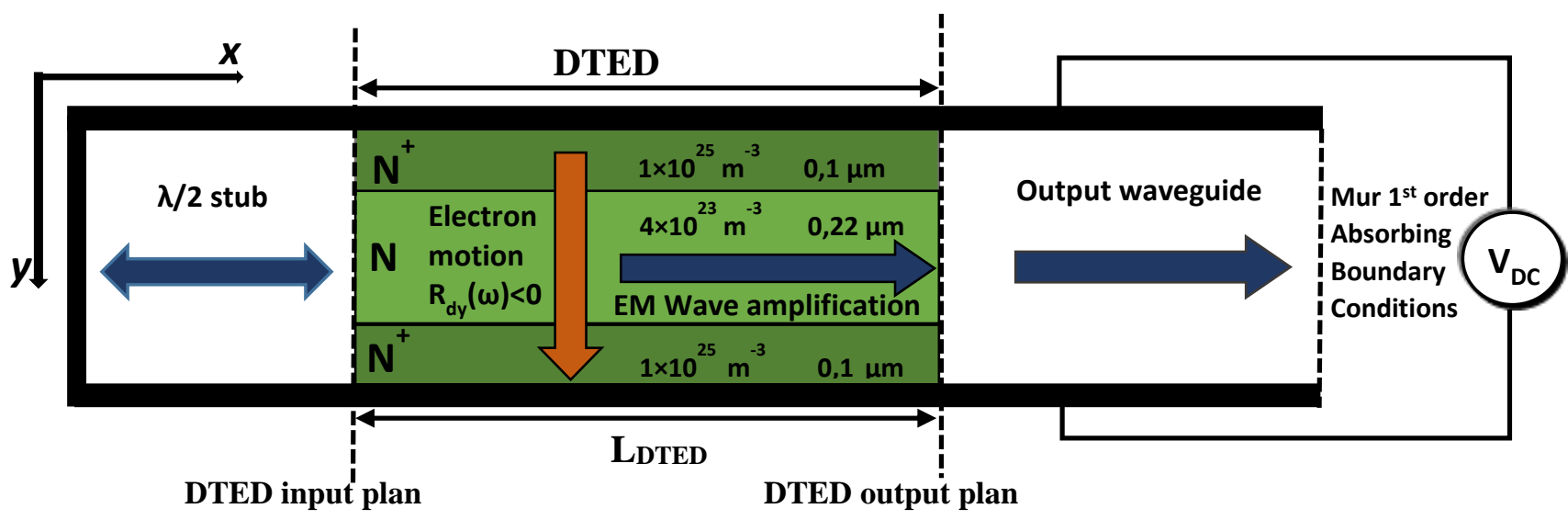

Figure 3: Parallel waveguide GaN THz DTED oscillator structure

4. DTED oscillator modelling

A realistic DTED oscillator modelling requires to account for the active device/passive load circuit interaction. Numerous load structures can be considered. Some seriously complicates the modelling. According to the DTED technological structure, the simplest way is to consider a parallel waveguide type passive load circuit behaving as a natural extension of the DTED structure (Fig. 3). Thus, a shorted stretch of planar waveguide or stub is connected to the DTED input plan. Its role is to impose an EM short circuit in the DTED input plan. Consequently, its length of $45 \mu \mathrm{m}$ corresponds to a half wavelength at the one $\mathrm{THz}$ expected fundamental frequency of the DTED RF operation. Note that in order to simplify the modelling, the stub is not DC isolated from the DTED terminals. This assumption is justified because the DC and transient modeling are independently solved. The output waveguide is a simple extension from the DTED terminals in which the propagation of a progressive EM wave is expected. Thus, absorbing boundary conditions are imposed in the oscillator output plan in order to avoid parasitic reflections [26]. This comes down to consider the oscillator output wave guide as matched to its characteristic impedance. Because of computer time limitation, its length must have been limited to $79 \mu \mathrm{m}$. The internal passive waveguide parts are considered as filled of a perfectly insulating material which permittivity is equal to that of $\mathrm{GaN}$. This allows for limiting the EM wave reflection resulting from the discontinuity effect at the DTED/output wave guide interface.

A correct DTED RF operation requires that, despite the unavoidable EM wave/electron transport $2 \mathrm{D}$ interaction, the electron RF operation remains mainly one-dimensional in the $y$ direction. From an EM point of view, this means that the propagation of a transverse electromagnetic wave (TEM) is expected in the whole oscillator structure.

In a preliminary study, the geometrical and technological parameters of the DTED N active zone are optimized. Thus, the electron transport through the epitaxial layers is analyzed in the lack of EM propagation effects. Thus, the device is considered as a classical vertical lumped structure and the electron transport as one-dimensional (1D). Consequently, this study can be performed by means of the simple time domain quasi-electrostatic/energy-momentum modeling under $\mathrm{CW}$ pure sine mode [14]. This allows determining the device DC and RF operating conditions leading to the best performance in terms of expected negative resistance level and associated RF power generation [14]. The optimum flat doping profile $\mathrm{GaN} \mathrm{N}^{+} \mathrm{NN}^{+}$TEDs operating at $1 \mathrm{THz}$ at $500 \mathrm{~K}$ has an $\mathrm{N}$ active zone thickness of $0,22 \mu \mathrm{m}$ with a doping level of $4 \times 10^{17} \mathrm{~cm}^{-3}$. The DTED length $\mathrm{L}_{\text {DTED }}$ is here $88 \mu \mathrm{m}$. All the results are here presented considering a length $\mathrm{L}_{\mathrm{z}}$ equal to $10 \mu \mathrm{m}$.

\subsection{DTED oscillator RF operation simulation}

DTED oscillator time domain simulations require the preliminary knowledge of an accurate spatial configuration of all the involved physical quantities. These initial solutions are obtained from simulations performed under DC steady-state operation. Because the electron transport only takes place in the $y$ transverse direction, the simple 1D quasielectrostatic/energy momentum modelling can be still used. Thus, for a DC bias voltage $\mathrm{V}_{\mathrm{DC}}$, the $\mathrm{EM}$ quantities are determined:

$$
E_{D C y}, H_{D C z}, J_{D C y}, \vec{E}_{R F}=\vec{H}_{R F}=0
$$

as well as the corresponding electron transport ones. Note that some of the DC evolutions have been incorporated for illustration in figure 5 .Then, the time-domain simulation is performed. The oscillation regime starts and grows from the weak numerical perturbations occurring at the DTED 
boundaries which behave as a kind of EM low power noise source of the order of $10^{-12} \mathrm{~W}$. Indeed, the transverse negative resistance effect is maximum at the lowest $E_{\mathrm{RFy}}$ levels [14]. The growing EM wave, propagating along the $x$ longitudinal direction, determines the transient phase of the order of 10 to $50 \mathrm{ps}$. The simulation is performed until stable RF waveforms are reached. Moreover, a long enough $\mathrm{CW}$ operation is simulated to allow a spectrum analysis by fast Fourier transform (FFT) with a sufficient frequency resolution. Thus, the required simulation duration is between 100 and 200 ps. Note that this paper is exclusively devoted to a DTED oscillator designed and DC biased to operate under near optimum RF conditions at one THz. Indeed, a great variety of unexpected RF operating mode can be pointed out (smothered, parametric, chaotic ...) depending on the oscillator DC and RF operating conditions as well as the DTED technological structure, particularly its length $\mathrm{L}_{\mathrm{DTED}}$ [10]. Their analysis present no interest in the present paper.

\subsection{DTED oscillator transient and CW operation}

The EM energy conservation equation expresses that, in a volume $\mathrm{v}$, the time variation of the internal energy (or power), including the contributions of the energy associated to the EM field $\mathrm{W}_{\mathrm{EH}}$ and the free electron kinetic energy $\mathrm{E}_{\mathrm{c}}$, is equal to the opposite of the EM energy transferred through the area $S$ surrounding the volume $\mathrm{v}$ :

$\iiint_{v} \frac{\partial W_{E H}}{\partial t} d v+\frac{\partial E_{C}}{\partial t}=-\iint_{S} \overrightarrow{P t g} d S$

$$
\text { with } \frac{\partial E_{c}}{\partial t}=\iiint_{v} \overrightarrow{J_{c}} \vec{E} d v
$$

$$
\text { and } \overrightarrow{P t g}=\vec{E} \Lambda \vec{H} \text { is the Poynting vector }
$$

Figure 3 illustrates the transient evolution of the DTED electron and output EM power.

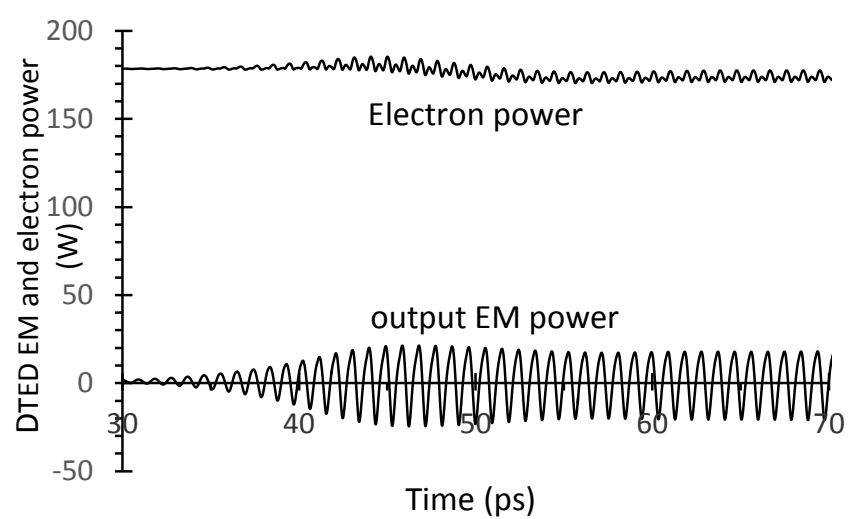

Figure 3: Transient electron and DTED output EM power evolutions $\left(\mathrm{L}_{\mathrm{DTED}}=88 \mu \mathrm{m}, \mathrm{V}_{\mathrm{DC}}=9 \mathrm{~V}\right)$.

It shows that, starting from the DC bias conditions, $30 \mathrm{ps}$ are necessary to reach EM fields of significant amplitude and the stable RF operation is reached after about 70 ps.

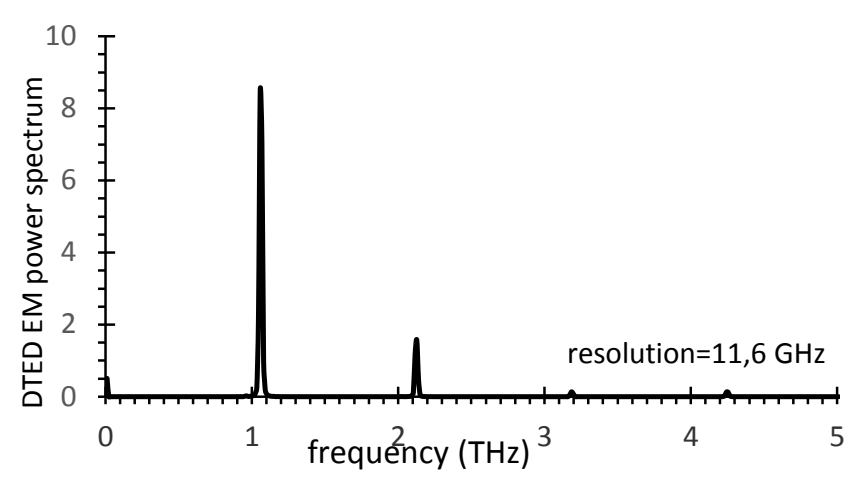

Figure 4: Frequency spectrum of output EM power under $\mathrm{CW}$ operation $\left(\mathrm{L}_{\mathrm{DTED}}=88 \mu \mathrm{m}, \mathrm{V}_{\mathrm{DC}}=9 \mathrm{~V}\right)$.

Under CW operation, the simulation is here continued on a duration of $100 \mathrm{ps}$ leading, for the Fourier analysis, to a frequency resolution of the order of $10 \mathrm{GHz}$. Figure 4 shows the spectrum of the output EM emitted power. Instead the DTED has been designed to operate at exactly $1 \mathrm{THz}$, the fundamental frequency line is near $1.05 \mathrm{THz}$. This result is globally consistent with the expected DTED CW operating mode and design. The additional harmonic lines results from the DTED intrinsic nonlinear behaviour. The amplitude of the second harmonic is not negligible and reaches about $20 \%$ of the fundamental one. Note the presence of low level third and fourth harmonic lines.

\subsection{DTED internal CW operating mode}

\subsubsection{Transverse operating mode}

Figure 5 illustrates the typical space-time one-dimensional physical quantities obtained under $\mathrm{CW}$ operation during a period $\mathrm{T}$, here close to one nanosecond. They are nearly 0,25 ps spaced out. Thus, they describe a complete RF cycle. They are the DC and RF electric field intensity and the electron density, velocity and energy along the $\mathrm{y}$ transverse direction near the device output interface $(x=130 \mu \mathrm{m})$ where the propagating $\mathrm{EM}$ field reaches its maximum magnitude allowing displaying in the most representative manner the involved physical phenomena.

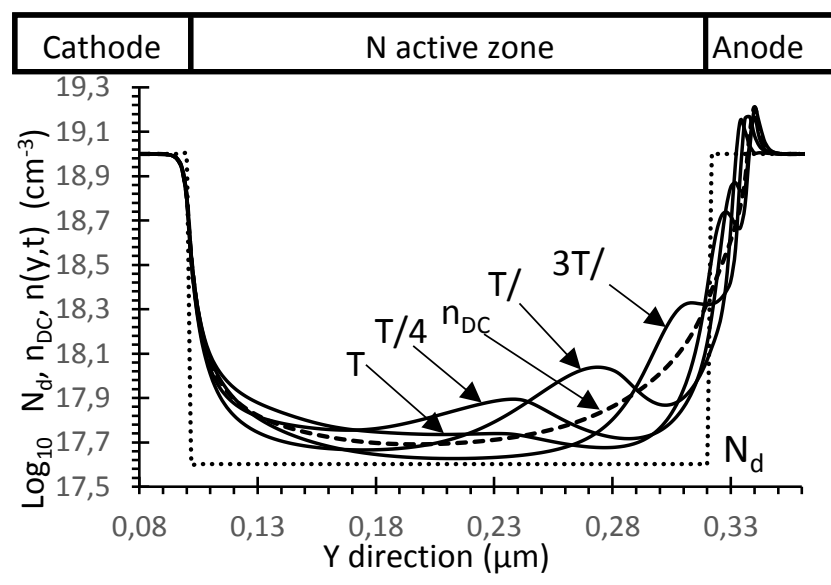




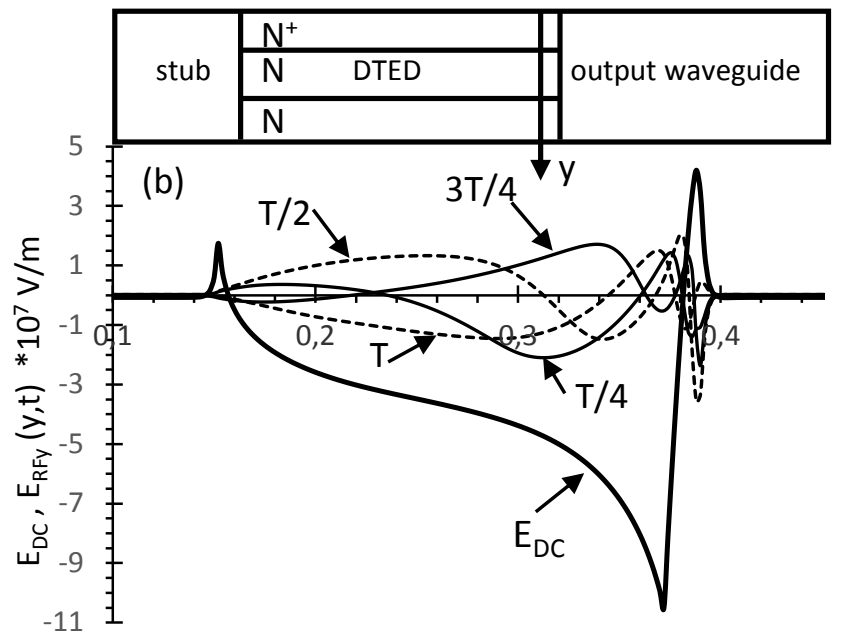

$\mathrm{Y}$ direction $(\mu \mathrm{m})$
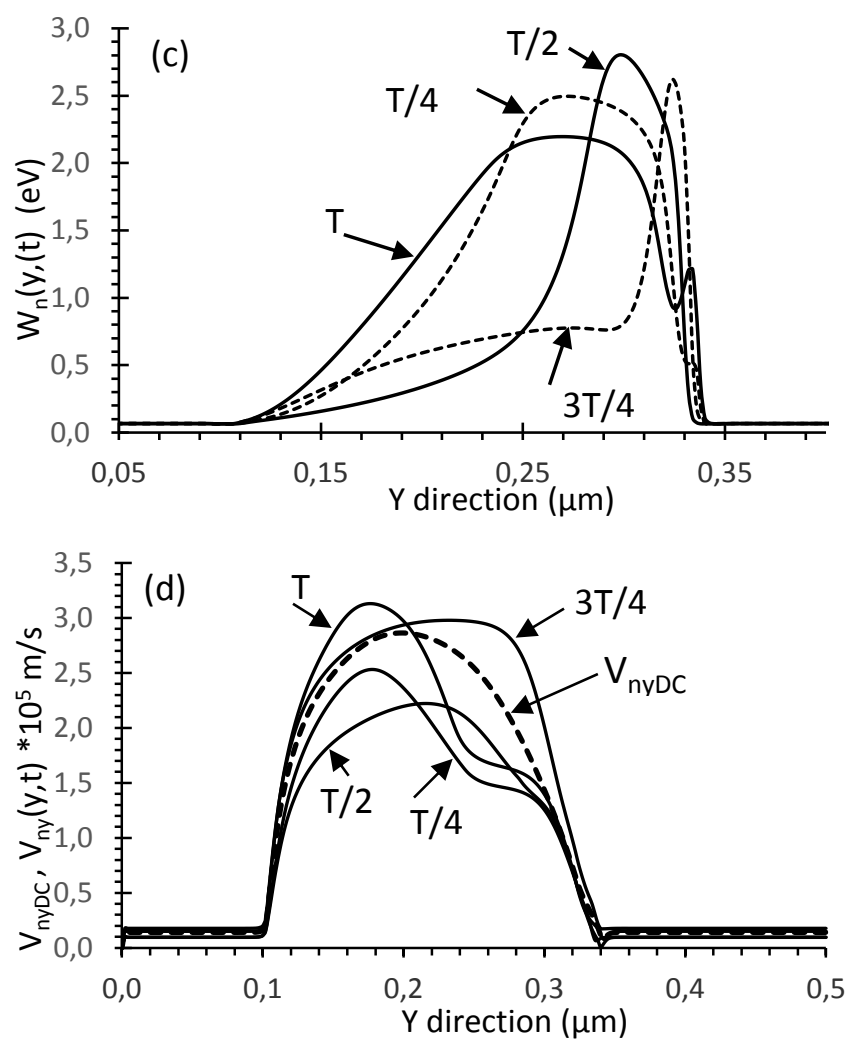

Figure 5: Y direction space-time evolutions of the transverse electron transport quantities over a RF cycle at $\mathrm{x}=130 \mu \mathrm{m}$.

The space-time evolutions show that the electrons, continuously injected from the forward biased $\mathrm{N}^{+}$cathode contact, gain energy under the action of the electric field. The electron energy relaxation effect tends to delay the electron heating. Consequently, the electrons keep a high mobility as they move in an already high electric field. This spatial transient effect yields the strong electron overvelocity $\left(v_{n}>v_{\text {peak }}\right)[24]$. When the GaN material energy threshold is reached, the electronic transfer phenomenon yields a decrease of the electron velocity leading to the electron accumulation layer formation. The local increase in electron density yields a strong space charge effect leading to a strong increase of the electric field intensity. Thus, all along the RF cycle, the electron accumulation layer is continuously reinforced and drifts at near saturation velocity under the action of the high electric field that she has herself contributed to create until it is collected by the anode contact at the end of the RF cycle. This RF operation is typically the expected electron accumulation layer and single transit time mode [24]. Following the simplified quasi-electrostatic approach and when stable $\mathrm{CW}$ operating mode is reached, Fourier analysis of the instantaneous y direction voltage and total current waveforms shows that the device presents a transverse negative resistance at the fixed operating frequency to the origin of the longitudinal EM wave amplification property.

\subsection{DTED oscillator RF operation EM analysis}

\subsubsection{EM propagation mode identification}

In 2D EM modeling and because of the Maxwell equation grouping, the EM propagation modes can be, in a generic manner, spilt in uncoupled transverse electric (TE) and transverse magnetic (TM) ones [25]. The DTED oscillator EM operation is fundamentally governed by the DTED DC bias condition that is the $\mathrm{y}$ direction $\mathrm{DC}$ electric field component $E_{D C y}$. Thus, only the $E_{R F x}, E_{R F y}$ and $H_{z}$ EM field components play a role in the oscillator RF operation while the $\mathrm{H}_{\mathrm{x}}, \mathrm{H}_{\mathrm{y}}$ and $\mathrm{E}_{\mathrm{z}}$ ones keep zero values. The analysis of the space-time EM fields allows to determine the EM propagation mode (figures 6 and 7). As an example, figures 6.a and 7.a shows the spatial evolutions of the magnitude of the $\mathrm{x}$ and $\mathrm{y}$ direction RF EM field components at the fundamental frequency in significant zones, which are respectively the region near the DTED output plan for the $\mathrm{x}$ direction and the region near the $\mathrm{N} / \mathrm{N}^{+}$anode interface, where the RF EM field reaches its maximum intensity.

The ratio between the respective magnitudes of the electric field components gives information to the type of EM wave propagation mode. In the stub and output waveguide, $\mathrm{E}_{\mathrm{RFx}}$ is logically zero. The related EM propagation mode is transverse electromagnetic (TEM or TM0). In the DTED region, the $E_{\mathrm{RFx}}$ amplitude is weak but not zero. Thus, from a strict EM point of view, The EM propagation mode is transverse magnetic (TM1). However, because of the electric component ratio $E_{\mathrm{RFx}} / \mathrm{E}_{\mathrm{RFy}}$, which is of the order of $1 \%$, the propagation mode can be considered as quasi-TEM. Thus, instead the waveguide dimensions, the TM1 mode propagates through the DTED region. Indeed, its cutoff frequency is lowered because of the flowing electron current [8]. 

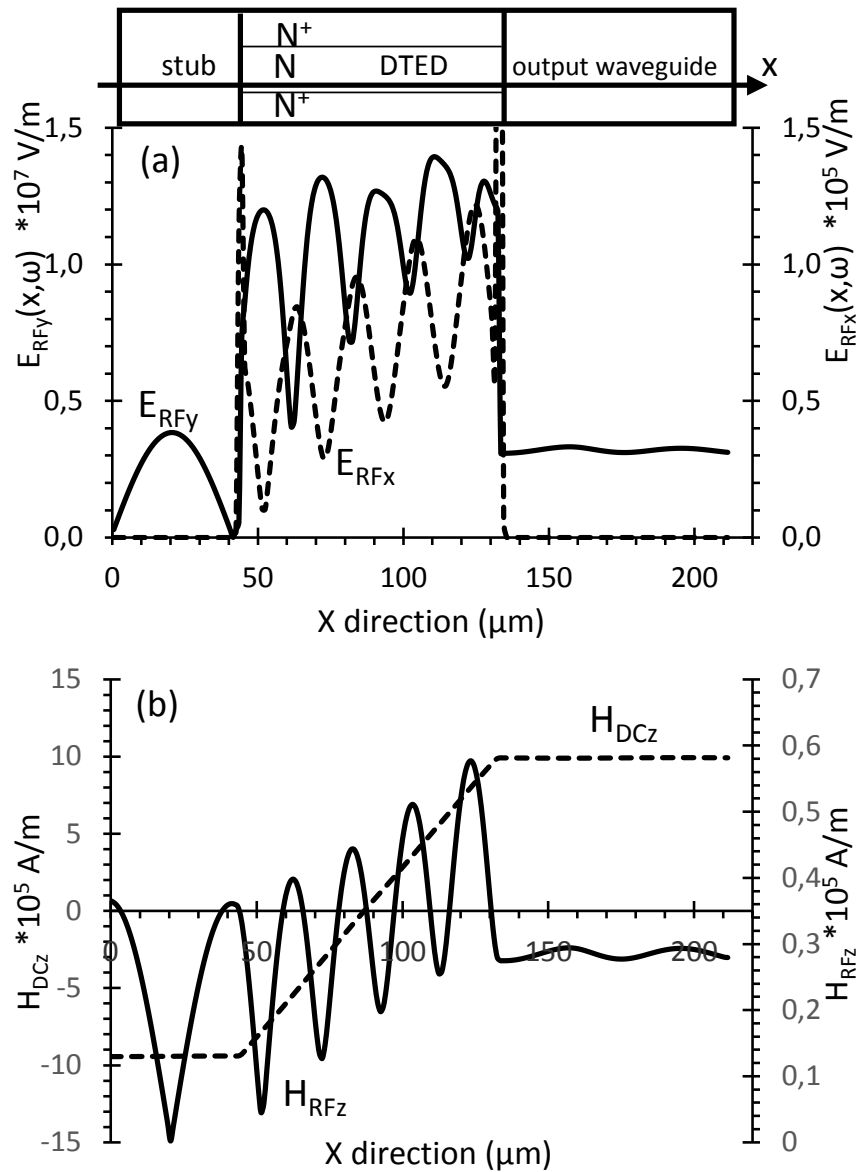

Figure 6: Spatial evolutions of the magnitude of the $x$ direction EM field components at the fundamental frequency at $\mathrm{y}=0,35 \mu \mathrm{m}$

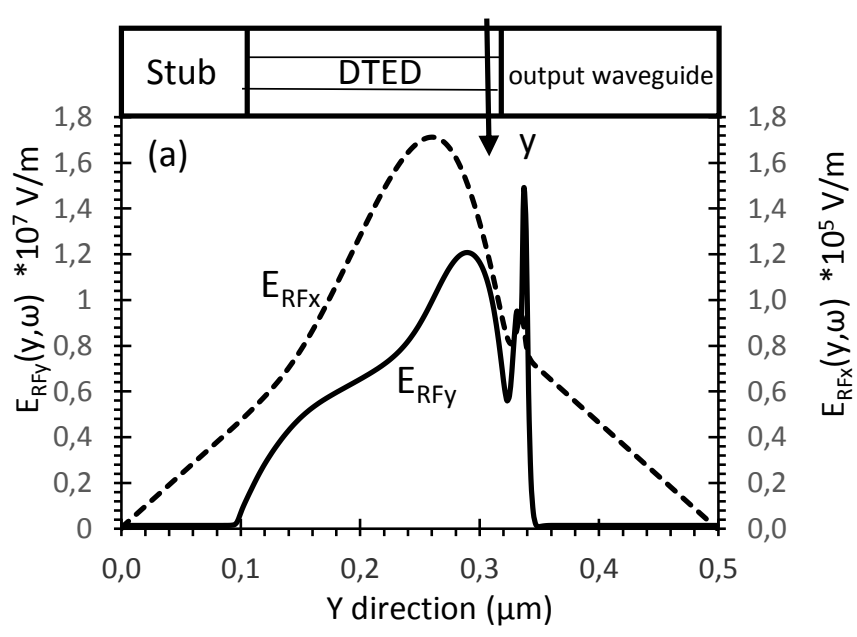

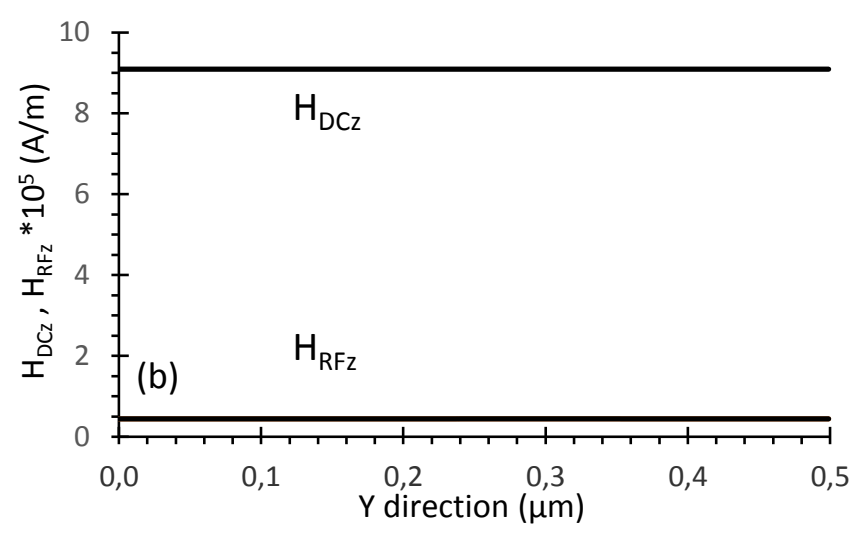

Figure 7: Spatial evolutions of the magnitude of the $y$ direction RF field components at the fundamental frequency at respectively at $\mathrm{x}=130 \mu \mathrm{m}$.

The DC electron current density $\mathrm{J}_{\mathrm{DCy}}$ is uniform in the DTED. Consequently, the $\mathrm{z}$ direction DC magnetic field component $\mathrm{H}_{\mathrm{DCz}}$ linearly varies in the $\mathrm{x}$ direction (figure 6.b) while remains unchanging in the y direction (figure 7.b). A strong discontinuity of the transverse electric and magnetic field components $\mathrm{E}_{\mathrm{RFy}}$ and $\mathrm{H}_{\mathrm{RFy}}$ is observed in the DTED/output waveguide interface. Indeed, the RF EM field generation is localized in a part of the electron space charge transit zone (see figure 16) while it is immediately uniformly distributed in the output waveguide because of the propagation of the TEM mode. Note that the present evolutions correspond to the zone where this effect is the most pronounced.

\subsubsection{Influence of $E_{R F x}$ on the lateral electron motion}

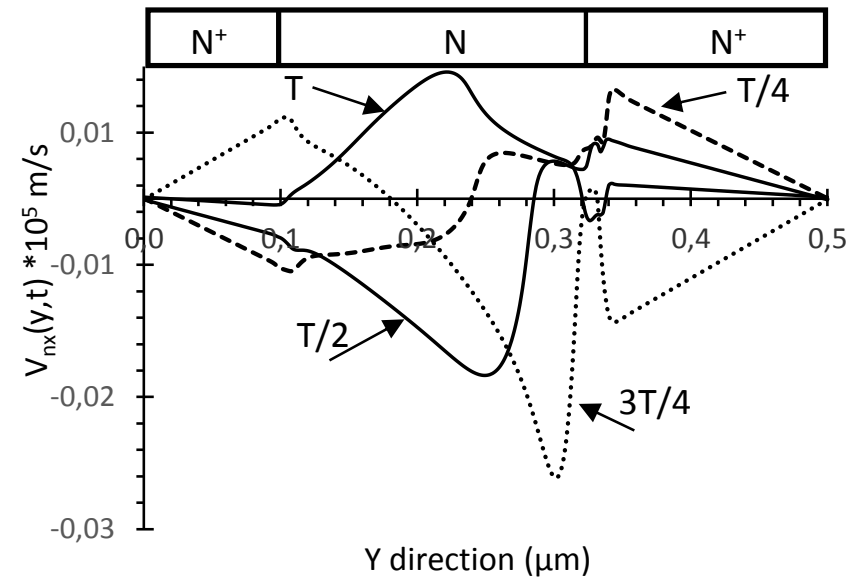

Figure 8: $\mathrm{Y}$ direction space-time evolution of the $\mathrm{x}$ direction component of the electron evolution at $y=130 \mu \mathrm{m}$ during a RF cycle.

Instead its low amplitude, the $\mathrm{x}$ direction electric field component $\mathrm{E}_{\mathrm{RFx}}$ yields a slight electron lateral motion in the DTED $\mathrm{N}$ active zone as illustrated in figure 8 showing the space-time evolutions of the $\mathrm{x}$ direction electron velocity $\mathrm{V}_{\mathrm{nx}}$. The local values of $\mathrm{V}_{\mathrm{nx}}$ can be compared with those of $\mathrm{V}_{\mathrm{ny}}$ (figure 5.d). The ratio $\mathrm{V}_{\mathrm{nx}} / \mathrm{V}_{\mathrm{ny}}$ is of the order of $1 \%$. Thus, the 
parasitic lateral electron motion does not fundamentally modify the DTED global RF operation. Moreover, this $\mathrm{x}$ direction motion is not unidirectional as compared with that in the $\mathrm{y}$ direction but appears to be a to-and-fro motion. However, its effect is not negligible. It slightly decreases the electron space charge transit time in the transverse direction. Thus, the DTED free running fundamental frequency of oscillations is lightly increased as observed in figure 4 . This feature would be taken into account in the design of the DTED technological structure.

\subsubsection{EM propagation regime}

\subsubsection{Longitudinal EM energy propagation}
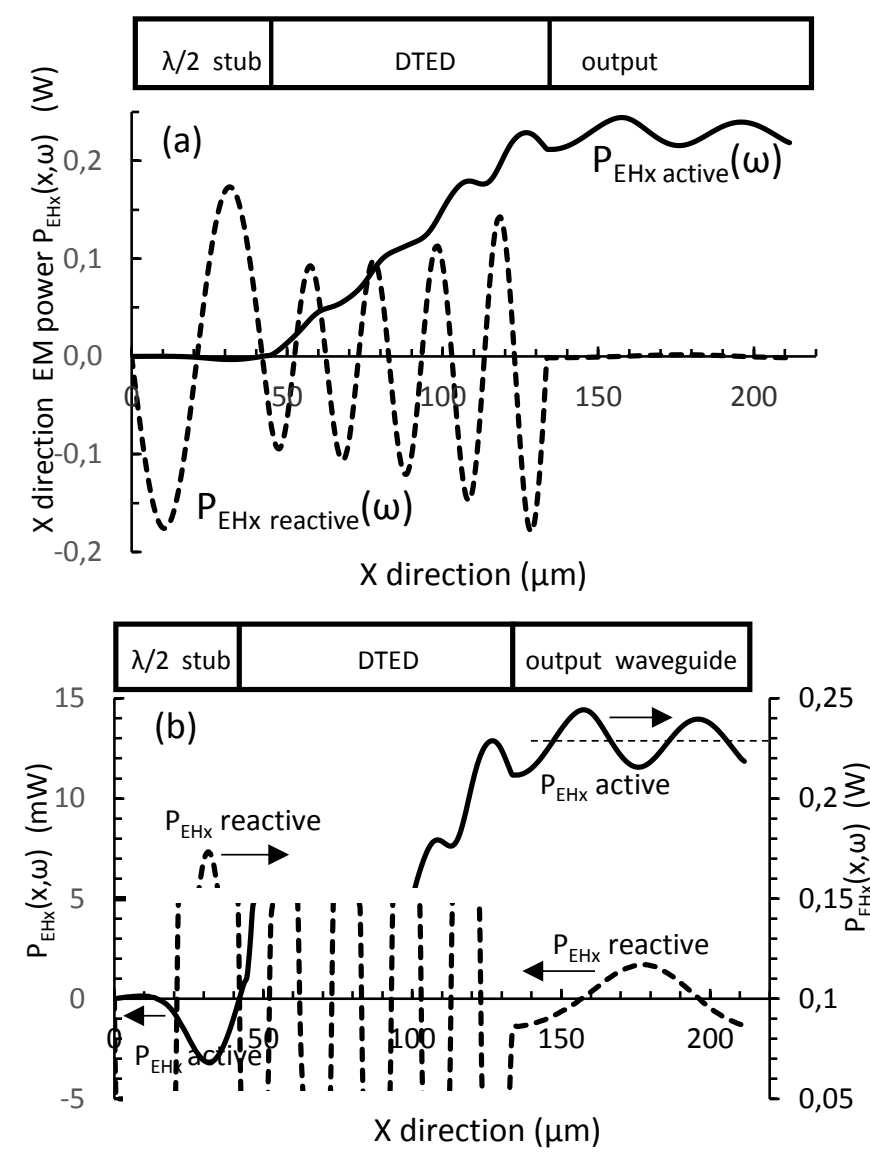

Figure 9: $X$ direction EM power $\mathrm{P}_{\mathrm{EHx}}$ through $\mathrm{S}_{\mathrm{yz}}$ area at the fundamental frequency. (a) full scale, (b) zoom view.

The EM power $\mathrm{P}_{\mathrm{EHx}}$ (or EM energy flux) delivered by the oscillator is the main functional quantity of interest for the circuit user. It can be simply calculated at a time $t$ by means of the $\mathrm{x}$ direction component of the Poynting vector flux $\operatorname{Ptg}_{\mathrm{x}}(\mathrm{x}, \mathrm{y}, \mathrm{t})$ through the $\mathrm{S}_{\mathrm{yz}}$ orientated transverse area for example at the boundary of the output waveguide:

$$
P_{E H x}(x, t)=\iint_{S_{y z}} \operatorname{Ptg}_{x}(y, x, t) d S=L_{z} \int_{0}^{L_{y}} \operatorname{Ptg}_{x} d y
$$

The EM propagation mode is pure or quasi-TEM all along the oscillator structure. The most significant EM field components are $\mathrm{E}_{\mathrm{y}}(\mathrm{y}, \mathrm{x}, \mathrm{t})$ and $\mathrm{H}_{\mathrm{z}}(\mathrm{y}, \mathrm{x}, \mathrm{t})$. Thus:

$$
\operatorname{Ptg}_{x}(y, x, t) \cong E_{y}(y, x, t) * H_{z}(y, x, t)
$$

The RF power $\mathrm{P}_{\mathrm{EHx}}$ only gives information about the amount and direction of convection of the EM energy propagating through the $\mathrm{S}_{\mathrm{yz}}$ area. More significant information can be obtained from the $\mathrm{x}$ direction evolution of $\mathrm{P}_{\mathrm{EHx}}$ and especially from its Fourier factorization based on the calculation of $\mathrm{E}_{\mathrm{y}}(\mathrm{y}, \mathrm{x}, \omega)$ and $\mathrm{H}_{\mathrm{z}}(\mathrm{y}, \mathrm{x}, \omega)$ :

$$
\operatorname{Ptg}_{x}(y, x, \omega) \cong\left\|E_{y}\right\|\left\|H_{z}\right\| e^{j\left(\operatorname{Arg} E_{y}-\operatorname{Arg} H_{z}\right)}
$$

Figure 9.a shows the space evolution, in the $\mathrm{x}$ propagation direction, of the active and reactive parts of the $\mathrm{x}$ direction EM power $\mathrm{P}_{\mathrm{EHx}}(\mathrm{x}, \omega)$ through the transverse area $\mathrm{S}_{\mathrm{yz}}$ at the fundamental frequency $\omega$ :

$$
\begin{gathered}
P_{E H x}(x, \omega)=\iint_{S_{y z}} \operatorname{Ptg}_{x}(y, x, \omega) d S=L_{z} \int_{0}^{L y} \operatorname{Ptg} g_{x} d y \\
\text { with } P_{E H x \text { active }}=\operatorname{Re}\left[P_{E H x}(x, \omega)\right] \text { is the active power, } \\
P_{E H x \text { reactive }}=\operatorname{Im}\left[P_{E H x}(x, \omega)\right] \text { is the reactive power }
\end{gathered}
$$

From a general point of view, the evolutions show that, except for a small portion of the stub, the active EM power $\mathrm{P}_{\mathrm{EHx}}$ is positive indicating that the EM energy globally propagates in a unidirectional manner in the positive $\mathrm{x}$ direction. Moreover, it allows identifying the EM operation specific to each portion of the oscillator.

In the stub region, $\mathrm{P}_{\mathrm{EHx}}$ is quasi only reactive. Indeed, the maximum active EM power remains lower than $0,25 \%$ of that of the corresponding reactive one as illustrated on figure 9.b zooming the $\mathrm{P}_{\mathrm{EHx}}$ evolution at EM low power level. So, the $\mathrm{E}_{\mathrm{y}}$ and $\mathrm{H}_{\mathrm{z}} \mathrm{EM}$ field components vibrate in quadrature in the time domain as well as in space as shown in figure 10. Thus, the stub fully fills its role. The propagating EM wave is of pure stationary type and is reflected under normal incidence on the metallic boundary interface. As expected, because of its half wavelength fixed dimension, the stub imposes a RF EM field of zero intensity at the DTED input transverse interface. Indeed, at a fixed x position, the transverse EM field component value does not depend on the position in the $y$ direction. 


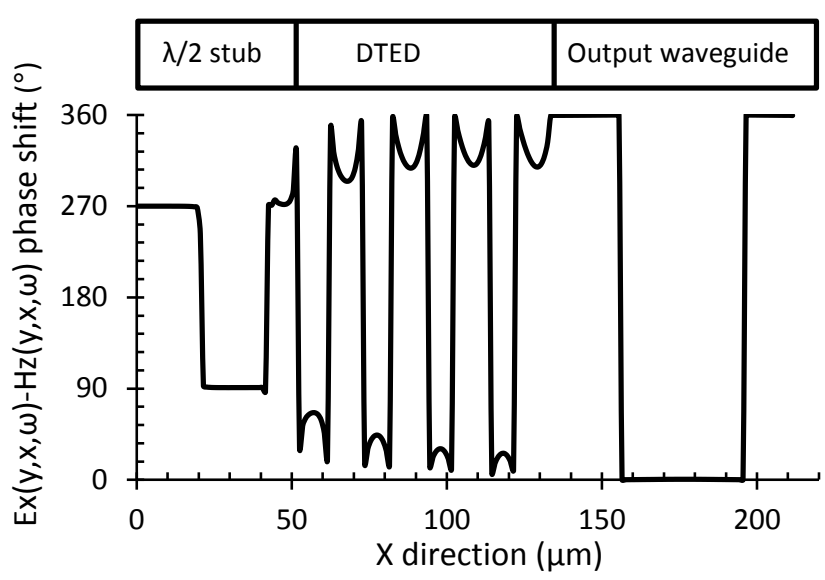

Figure 10: $\mathrm{X}$ direction space evolution of the phase shift between the EM field components $\mathrm{E}_{\mathrm{y}}$ and $\mathrm{H}_{\mathrm{z}}$ at $\mathrm{y}=0,35 \mu \mathrm{m}$.

In the DTED region, $\mathrm{P}_{\mathrm{EHx}}$ is complex. The $\mathrm{E}_{\mathrm{y}}$ and $\mathrm{H}_{\mathrm{z}}$ components do not vibrate in phase. The EM wave regime is of half-stationary type. The EM energy flux transported by the wave corresponds to the real part of $\mathrm{P}_{\mathrm{EHx}}$. It nearly linearly increases all along the DTED region under the action of the amplification phenomenon resulting from the free electron/EM wave interaction.

In the output waveguide, $\mathrm{P}_{\mathrm{EHx}}$ is quasi only active. The $\mathrm{E}_{\mathrm{y}}$ and $\mathrm{H}_{\mathrm{z}}$ components are in phase. The EM propagation regime is progressive in the $\mathrm{Y}$ direction.

In a pure dielectric planar waveguide in which propagates a CW monochromatic EM wave, the active EM power is normally constant all along the waveguide. By contrast, a ripple of the active $\mathrm{P}_{\mathrm{EHx}}$ is observed in both the DTED and output waveguide regions. This feature can result from the combination of several phenomena which can be gathered in two main categories:

- The non-linearity of the electron/EM field interaction.

-The influence of the different interfaces.

The relative influence of each of these phenomena cannot be directly and independently evaluated from the space-time EM field but from their analysis.

\subsubsection{Transverse EM energy propagation}

Instead the weakness of the $\mathrm{y}$ direction waveguide dimension $\left(l_{y}=0,5 \mu \mathrm{m}\right)$, EM energy propagation occurs in the transverse direction. The EM power $\mathrm{P}_{\mathrm{EHy}}$ through the orientated surface $\mathrm{S}_{\Delta \mathrm{xLz}}$ can be evaluated from the EM field components $\mathrm{E}_{\mathrm{x}}(\mathrm{y}, \mathrm{x}, \mathrm{t})$ and $\mathrm{H}_{\mathrm{z}}(\mathrm{y}, \mathrm{x}, \mathrm{t})$ in a slice at a position $\mathrm{x}$ of the oscillator:

$$
\begin{gathered}
P_{E H y}(y, t)=\iint_{S_{\Delta x L z}} \operatorname{Ptg}_{y}(y, x, t) d S \\
\text { with } \operatorname{Ptg}_{y}(y, x, t) \approx-E_{x} H_{z}
\end{gathered}
$$

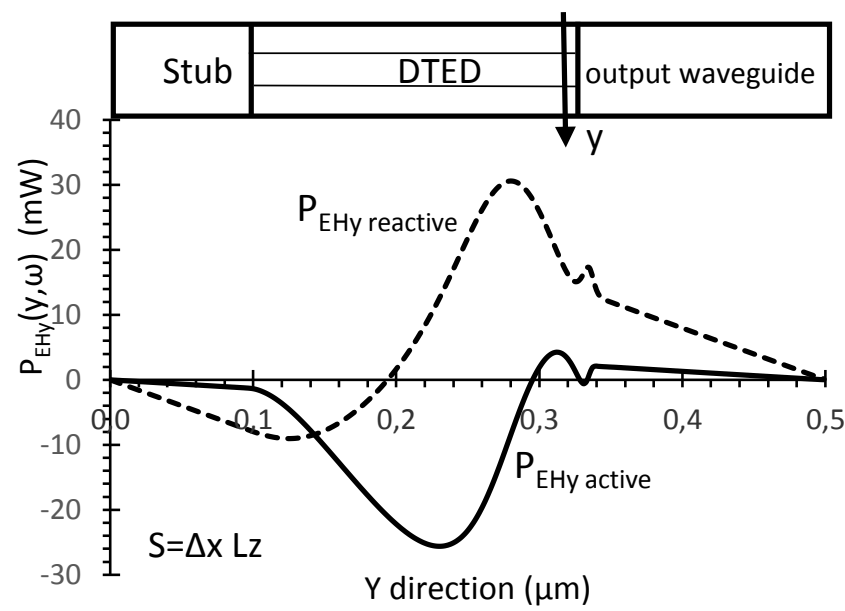

Figure 11: Space evolution of the y direction EM power $\mathrm{P}_{\mathrm{EHy}}$ at the fundamental frequency $\omega$ in a slice at $x=130 \mu \mathrm{m}$.

Figure 11 shows a typical space evolution of $\mathrm{P}_{\mathrm{EHy}}$, issued from its Fourier analysis at the fundamental frequency $\omega$, in a slice localized near the DTED output interface at $\mathrm{x}=130 \mu \mathrm{m}$. $\mathrm{P}_{\mathrm{EHy}}(\mathrm{y}, \omega)$ gives information to the influence of the EM field component $E_{\mathrm{RFx}}$. It yields, in the DTED $\mathrm{N}$ active zone, an active EM energy flux in the y opposite direction.

\subsubsection{Influence of the non-linear RF operation}

\subsubsection{Influence of the transverse electric field amplitude}

The electron motion mainly occurs in the transverse $y$ direction. It is governed by the electric field component $\mathrm{E}_{\mathrm{y}}$. This local interaction is non-linear. A way to globally evaluate the influence of this phenomenon on the DTED RF operation is to consider the evolution of the intrinsic RF emitted power $\mathrm{P}_{\mathrm{RF}}$ in a DTED elementary slice in the lack of EM propagation effect. Following this assumption, the slice can be considered as a simple $\mathrm{N}^{+} \mathrm{NN}^{+}$vertical TED. The $\mathrm{P}_{\mathrm{RF}}$ power can be obtained from the one-dimensional timedomain quasi-electrostatic/energy-momentum transport model allowing for determining the $\mathrm{y}$ direction total current/voltage instantaneous relation [14]:

$$
\begin{gathered}
V(t)=-\int_{0}^{L_{y}} E(y, t) d y \\
J_{t}(t)=J_{c}(y, t)+\varepsilon \frac{\partial E(y, t)}{\partial t}
\end{gathered}
$$

CW pure sine simulation associated to Fourier analysis yields the evolution of the RF emitted power and even RF negative resistance level as a function of the RF voltage at 1 $\mathrm{THz}$ as shown in figure 12 , here for an arbitrary area $\mathrm{S}_{\mathrm{xz}}$ of $10^{-10} \mathrm{~m}^{2}$. 


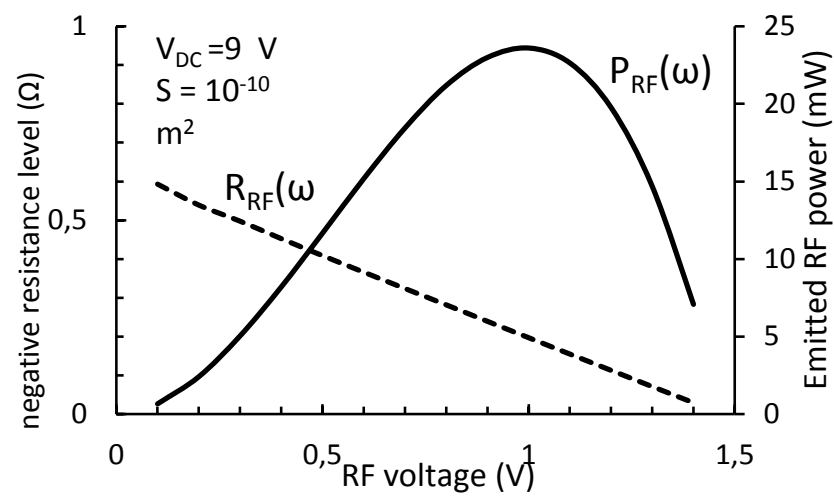

Figure 12: Intrinsic RF emitted power and negative resistance level in an elementary slice of the DTED as a function of the RF voltage.

The RF emitted power increases nearly linearly up to reach a peak value above which it decreases roughly for reaching negative values corresponding to a loss RF operation.

Figure 13 now shows, for the DTED oscillator, the space evolution, in the $\mathrm{x}$ propagation direction, of the amplitude of the transverse RF voltage $V_{R F y}(x, \omega)$ at the fundamental frequency $\omega$ obtained from the Fourier analysis of its instantaneous value:

$$
V_{R F y}(x, t)=-\int_{0}^{L_{y}} E_{R F y}(y, x, t) d y
$$

The RF voltage $\mathrm{V}_{\mathrm{RFy}}(\mathrm{x}, \omega)$ logically presents a peak value in the middle of the $\lambda / 2$ long stub region as well as zero values at its boundaries. In the DTED region, $\mathrm{V}_{\mathrm{RFy}}(\mathrm{x}, \omega)$ presents a large and regular ripple but in average increases nearly linearly. Its values consistently range with those of the elementary slice (figure 12). At the DTED/output waveguide interface, $\mathrm{V}_{\mathrm{RFy}}(\mathrm{x}, \omega)$ reaches a maximum value of the order of $1,5 \mathrm{~V}$ and thus mainly remains lower than the range of values leading to a loss RF operation. Thus, according to the DC and RF operating conditions, the DTED region length appears in a first approximation optimum.

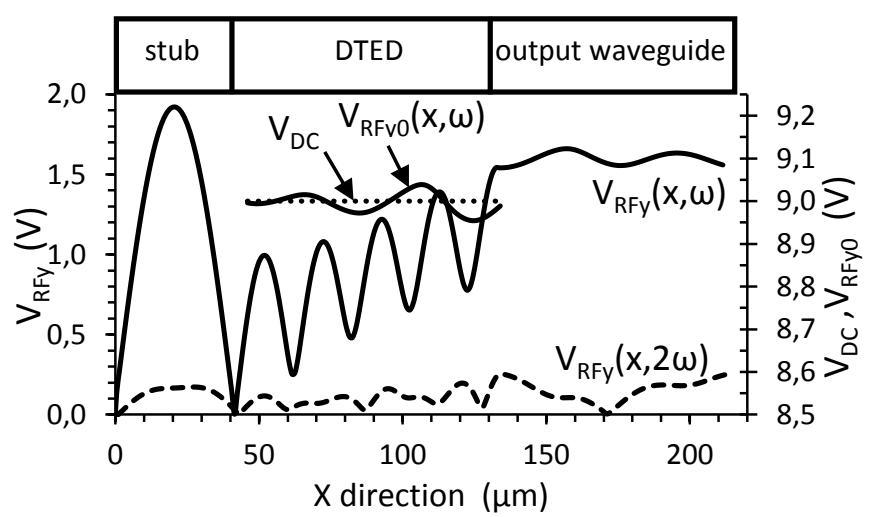

Figure 13: $\mathrm{X}$ direction evolution of the mean and RF voltage $\mathrm{V}_{\mathrm{RFy}}$ and DC bias voltage $\mathrm{V}_{\mathrm{DC}}$
This assumption can be refined by analyzing the RF properties of the oscillator split in transverse slice of thickness $\Delta \mathrm{x}$ (figure 1). The EM energy flux through the closed area surrounding a slice of volume $\Delta \mathrm{xL}_{\mathrm{y}} \mathrm{L}_{\mathrm{z}}$ and located in $\mathrm{x}$ position is:

$$
\begin{aligned}
P_{E H_{\text {Slice } x}}(x, t) & =\iint_{S_{\Delta x L_{y} L_{z}}} \overrightarrow{\operatorname{Ptg}}(y, x, t) d S \\
& =\Delta x L_{z}\left[P_{E H x}\left(x+\frac{\Delta x}{2}\right)-P_{E H x}\left(x-\frac{\Delta x}{2}\right)\right]
\end{aligned}
$$

Figure 14 presents the space evolution in the $\mathrm{x}$ direction of the active and reactive parts of $P_{E H_{\text {Slicex }}}$ at the fundamental frequency.

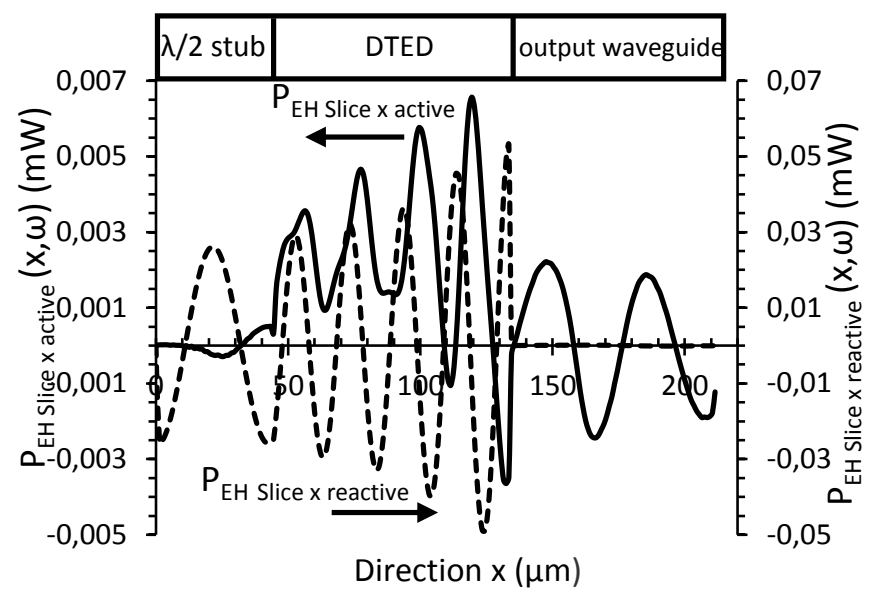

Figure 14: $\mathrm{X}$ direction evolution of the active and reactive EM power at the fundamental frequency $\omega$ in a transverse slice of the oscillator.

A positive value of the slice $\mathrm{x}$ active power indicates that the slice globally contributes to the RF EM energy flux generation. This result demonstrates that it is globally the case in the DTED active semiconductor region except two zones of weak thickness near the DTED/output wave guide interface where the active power becomes negative. Indeed, in these regions, the amplitude of the $\mathrm{y}$ direction RF electric field component $E_{\mathrm{RFy}}$ becomes so high that the transverse electron motion is no more the expected accumulation layer and transit RF operating mode. This two zones logically correspond to those where the RF voltage $V_{R F y}(x, \omega)$ is of the order of 1,5 V (figure 13). Thus, accounting for the DTED DC bias condition, the DTED length must not be more important. In a planar waveguide in which propagates a $\mathrm{CW}$ monochromatic TEM wave, the active EM power in the propagation direction through an orientated transverse surface is constant and consequently zero when considering a slice of the waveguide. In the oscillator output waveguide, the $\mathrm{x}$ direction evolution of active EM power $\mathrm{P}_{\mathrm{EHx}}$ in a slice is far to be constant. Indeed, it must be considered that the EM wave, coming from the DTED output interface acting as the EM wave source, has not reach its stable TEM waveform.

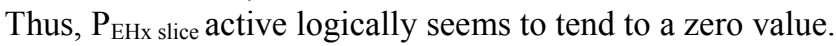




\subsubsection{Influence of harmonic EM fields}

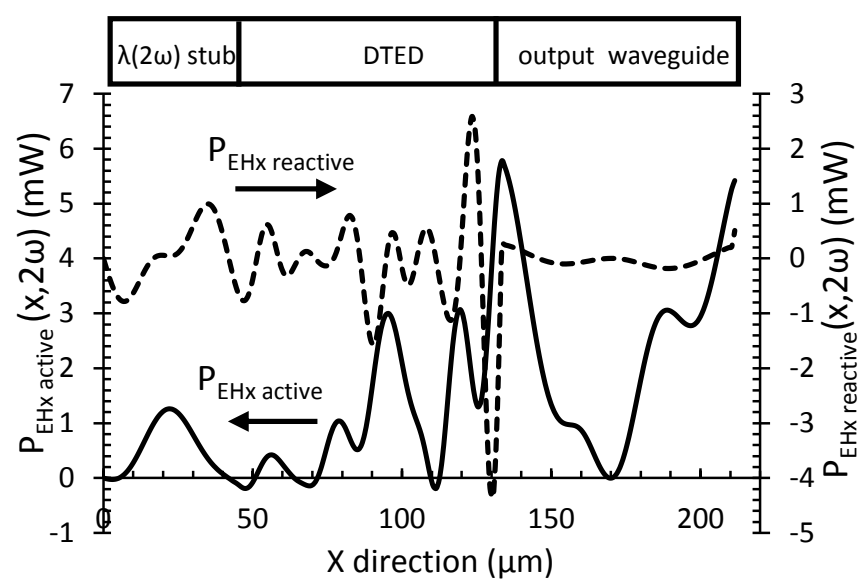

Figure 15: $\mathrm{X}$ direction complex EM power through $\mathrm{S}_{\mathrm{yz}}$ area at the second harmonic frequency.

The DTED RF operation is intrinsically nonlinear. Consequently, harmonic EM field components are generated. They are superimposed with the fundamental frequency one and are consequently able to influence the global DTED RF operation. Because of the present passive load circuit structure, they are neither controlled nor filtered. Figure 15 shows the $\mathrm{x}$ direction EM energy flux $\mathrm{P}_{\mathrm{EHx}}$ through the $\mathrm{S}_{\mathrm{yz}}$ area at the second harmonic frequency. From a general point of view, the involved EM power levels appear non negligible but clearly lower than those at the fundamental frequency (figure 9.a). Thus, the global DTED RF operation as expected remains mainly governed by the physical phenomena occurring at $\omega$. This is obvious when the transverse RF voltage evolutions are compared (figure 13). The stub is one spatial wavelength long at the second harmonic. It well imposed an EM short in the DTED input plan. The active EM energy flux globally increases in the DTED region but in an irregular manner.

\subsubsection{Self-bias effect}

The DTED nonlinear RF operation also appears in the selfbias effect. Figure 13 compares the $\mathrm{x}$ direction evolution of the DTED DC bias $\mathrm{V}_{\mathrm{DC}}$ and mean value $\mathrm{V}_{\mathrm{RFy}}$ of $\mathrm{V}_{\mathrm{RFy}}$ at the fundamental frequency $\omega$. The difference between the two quantities follows the amplification of the transverse EM field and reach a peak to peak amplitude of about $100 \mathrm{mV}$. This effect results from the RF to DC EM energy conversion.

\subsubsection{Influence of the interfaces}

\subsubsection{EM wave refection at the oscillator output boundary}

A wave of TEM type propagates through the output waveguide. Consequently, the direction of propagation of the EM wave is perpendicular to the oscillator transverse planar wave guide structure. In this case, the efficiency of the first order Mur absorbing boundary condition is maximum leading to the minimum EM energy reflection at the output wave guide boundary interface which can be as low as $10^{-4}$ [2526]. So, the contribution of this phenomenon to the active $\mathrm{P}_{\mathrm{EHx}}$ is certainly negligible in comparison with the other ones.

\subsubsection{EM wave reflection at the DTED/output waveguide interface}

The progressive EM wave reflection at the DTED/output waveguide interface generates a regressive wave propagating towards the DTED input plan. The latter is able to be amplified. This effect is certainly the most one of influence in the DTED RF operation. The progressive and regressive EM waves superimpose and cannot be distinguished from each other. Their ratio determines the ripple amplitude observed in the evolution of the EM power $\mathrm{P}_{\mathrm{EHx}}$ (figure 9.a).

\subsubsection{EM field propagation in the output wave guide}

The DTED/output waveguide interface constitutes the boundary between the EM field source and the propagation region. In the output waveguide, the transverse EM power $\mathrm{P}_{\mathrm{EHx}}(\mathrm{x}, \omega)$ presents a ripple of decreasing amplitude (figure 9.b). Indeed, because of its non-long enough dimension, the output waveguide behaves as a transition zone in which the propagating EM field has not yet reach the TEM mode stable waveform. Thus, $\mathrm{P}_{\mathrm{EHx}}(\mathrm{x}, \omega)$ seems to converge towards a constant value of the order of $230 \mathrm{~mW}$.

\subsubsection{DTED to output waveguide EM energy transfert}

The EM energy transfer from the DTED to the output waveguide is a key factor determining the oscillator performance. It cannot be optimized following the method based on the functional impedance matching concept classically used by the microwave circuit designers. In the output waveguide, at a far enough distance from the DTED/output waveguide interface, the stable waveforms of $\mathrm{E}_{\mathrm{y}}$ and $\mathrm{H}_{\mathrm{z}}$ allow to define constant functional quantities related to the EM power and propagation mode impedance. In the DTED, these quantities locally varies and can be positive as well as negative.

The EM power $\mathrm{P}_{\mathrm{EHxy}}$ through the surface surrounding an elementary volume $\mathrm{v}=\Delta \mathrm{x} \Delta \mathrm{y} \mathrm{Lz}$ is:

$$
\begin{aligned}
& P_{E H_{x y}}(y, x, t)=\iint_{S_{\Delta x \Delta y L z}} \overrightarrow{P t g}(y, x, t) d S \\
= & \Delta x L_{z}\left[P_{E H x}\left(x+\frac{\Delta x}{2}, t\right)-P_{E H x}\left(x-\frac{\Delta x}{2}, t\right)\right] \\
+ & \Delta y L_{z}\left[P_{E H y}\left(y+\frac{\Delta y}{2}, t\right)-P_{E H y}\left(y-\frac{\Delta y}{2}, t\right)\right]
\end{aligned}
$$

Significant information about the DTED internal RF EM behavior can be obtained from its Fourier factorization. Figure 16 shows the y direction spatial evolution $\mathrm{P}_{\mathrm{EHxy}}(\mathrm{y}, \omega)$ 
at the fundamental frequency at a position $\mathrm{x}$ close to the DTED output interface.

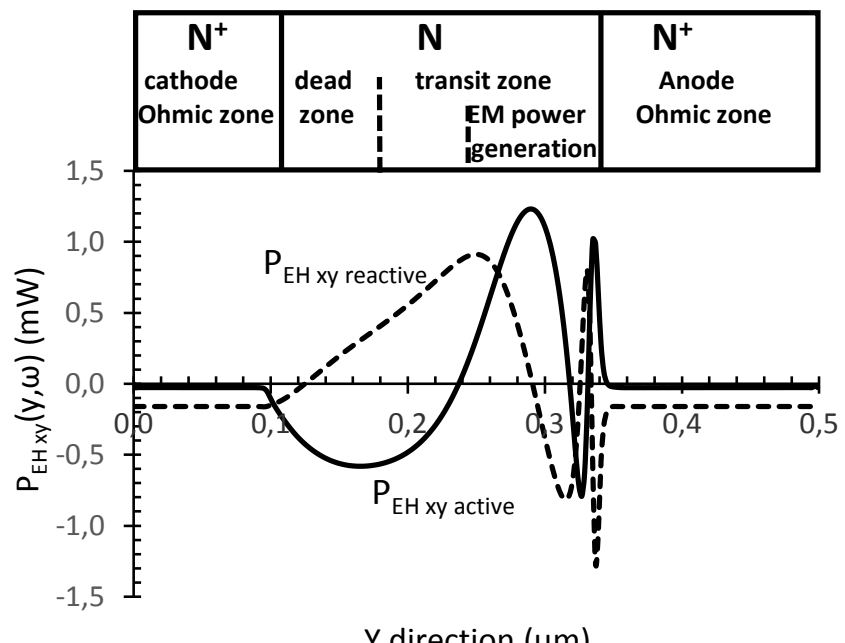

Figure 16: $\mathrm{Y}$ direction spatial evolution of the local EM power at the fundamental frequency $\omega$ at $x=130 \mu \mathrm{m}$

The cathode and anode ohmic zones behaves as RF loss regions. Indeed, the slightly negative value of the local active EM power indicates that the RF EM field provides EM energy to the drifting electrons. The $\mathrm{N}$ active region split in two subzones. In the first one, $\mathrm{P}_{\mathrm{EHxy}}$ active is clearly negative. The first one corresponds to the "dead zone" where the electrons gain the threshold energy from the electric field leading to the inter-valley transfer phenomenon and electron accumulation layer development. The second zone corresponds to the transit zone where the drifting electron layer contributes to the RF EM energy generation. However, only the zone where $\mathrm{P}_{\mathrm{EHxy}}$ active is positive really contributes to the EM power generation. Note that the sudden space variations at the $\mathrm{N}$ active zone $/ \mathrm{N}^{+}$cathode interface result from the strong modulation of the high field depleted zone width under the action of the $y$ direction RF EM electric field component $E_{R F y}$.

It remains possible to evaluate a coupling efficiency based on the evaluation of the ratio between the DTED output power and the power propagating through the output waveguide boundary. Note that this concept does not provide optimization rules usable for the circuit designer. The asymptotic value of the transverse $\mathrm{EM}$ power $\mathrm{P}_{\mathrm{EHx}}$ in the output waveguide which is of the order of $230 \mathrm{~mW}$ (figure 9.b). That in the vicinity of the DTED output interface is close to this value. Thus, we can conclude that, accounting for the DTED length and DC and RF operation, the transfer of EM energy from the DTED to the output waveguide is nearly optimum.

\subsubsection{EM field/free electron energy transfer}

The present modeling only account for the transfer of energy between the EM field and the free electron. It is expressed from the local instantaneous values of the electric field $\vec{E}(y, x, t)$ and the electron current density $\overrightarrow{J_{c}}(y, x, t)$ by means of the electron power $P_{J_{C} E}$ in an elementary volume $\mathrm{v}(\mathrm{y}, \mathrm{x})$ :

$$
\begin{gathered}
P_{J_{c} E}=\iiint_{v} \overrightarrow{J_{c}} \vec{E} d v=\iiint_{v} J_{c x} E_{x}+J_{c y} E_{y} d v \\
\text { with } v=\Delta x \Delta y L z
\end{gathered}
$$

Figure 17 details the different contributions, issued from the Fourier factorization, of the electron $\mathrm{P}_{\mathrm{JcE}}$ that are the DC one and RF ones at the fundamental frequency under CW RF operation.
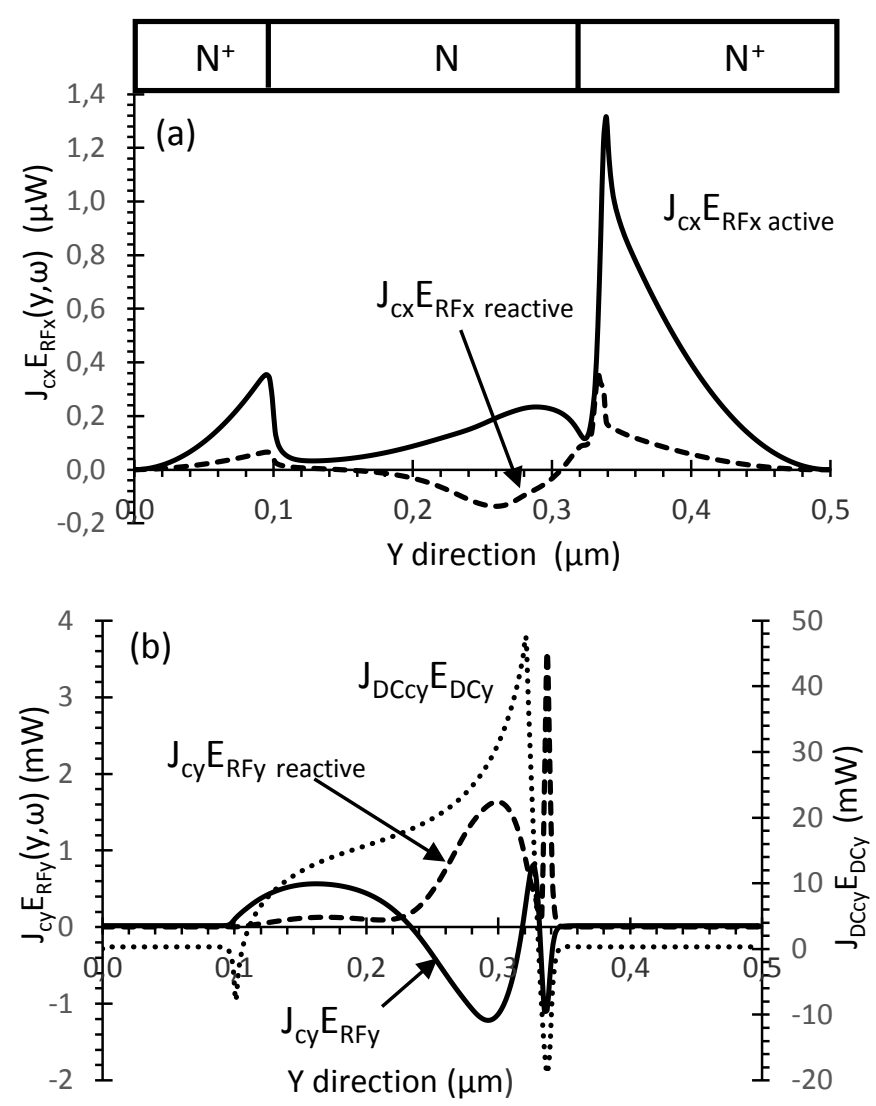

Figure 17: Y direction spatial evolution of the different terms constituting the electron power at the fundamental frequency $\omega$ at $x=130 \mu \mathrm{m}$

The usable EM power delivered by the oscillator only results from the EM energy propagation in the $\mathrm{x}$ direction. Consequently, the unavoidable electron drift involved by the $x$ direction electric field component fully contributes to RF losses. Figure 17.a clearly shows that the RF losses in the $\mathrm{x}$ direction mainly peak in the anode ohmic zone adjacent to the $\mathrm{N}$ active region. They present values of lesser importance in the high field region of the $\mathrm{N}$ active zone and in the cathode ohmic zone. 
Most of the energy absorbed by the electrons results from their $y$ direction motion imposed by the DTED DC bias conditions as shown in figure 17.b. The DC power follows the spatial evolution of the DC electric field component. The spatial evolution of the electron active RF power is typical of the RF behavior imposed by the RF electric field. In the $\mathrm{N}$ zone, the active electron power is positive where the electrons have to gain the threshold energy from the electric field. It is negative where the electron space charge drifts and contributes to the RF EM field generation. The electron reactive power is positive all along the $\mathrm{N}$ active zone traducing an inductive effect of the electron motion. It is not typical of the DTED global reactive behavior because it is in completion with the capacitive behavior resulting from the electron depletion of the high field $\mathrm{N}$ zone.

\section{Conclusi on}

A theoretical study devoted to the analysis of the CW operation of a planar waveguide $\mathrm{THz}$ oscillator based on a distributed transferred electron device has been presented in this paper. It has been performed by means of a $2 \mathrm{D}$ timedomain numerical physical modelling. This one is based on the consistent solution of the Maxwell equations for the description of the electromagnetic phenomena and the energy-momentum type macroscopic equations for the free electron transport. Most of the features of the complex oscillator RF operation have been considered following electromagnetic approach. They are the DTED internal RF operation, the EM wave propagation conditions, the influence of the nonlinear phenomena ... It is demonstrated that, on the basis of the near optimum oscillator technological parameters and DC bias conditions, the present oscillator operates as expected as well as form the EM point of view as from the electron transport one. However, a lot of work is still to be done. They must be for example extended to the study of the influence of the DTED length LDTED $_{\text {and the DTED DC bias }}$ conditions. Moreover, they could for example concern the DTED technological structure and the use of differentiated $\mathrm{N}$ active zone doping profile or the investigation of different RF operating modes as the electron accumulation layer and multi-transit one. In order to make up for the thermal limitation of the GaN semiconductor material, the use of an indium phosphide (InP) DTED could be considered. The difficulty to overcome would be in this case the electronic limitation. Another step could concern the coupling of the DTED to a different passive load circuit for example based on plasmonic waveguide technology. All the expected potential improvement could justify the investment in a monolithic technological realization

\section{Acknowledgements}

The author would like to thank J.-F. Legier for helpful discussions.

\section{References}

[1] H. Eisele, Fundamental solid-state terahertz sources, R. E. Miles, Ed. New-york: Springer-Verlag, 2007, pp. 69-88.

[2] C. Dalle," Theoretical investigation of THz GaN mesa Transferred-Electron Device by means of time domain energy/momentum modeling", IEEE Trans. Electron Devices, Vol. ED-55, n6, pp.1563-1567, December 2012.

[3] V. Gruzinskis, P. Shiktorov, E. Starikov, J. H. Zhao, "Comparative study of 200-300 GHz microwave power generation in GaN TEDs by the Monte Carlo technique", Semicond. Sci. Technol, 16, 798, 2001.

[4] S. Garcia, I. Iniguez-de-la-Torre, S. Perez, J. Mateos, T. Gonzalez, "Numerical study of sub-millimeter Gunn oscillations in InP and GaN vertical diodes: dependence on bias, dopin, and length", J. Applied Physics, 114, 074503, 2013.

[5] Z. C. Huang, R. Goldberg, J. C. Chen, Youdou Zheng, D. Brent Mott, P. Shu, "Direct observation of transferred-electron effect in GaN", App. Phys. Lett., 67(19), pp. 2825-2826, 6 november 1995.

[6] O. Yilmazoglu, K. Mutamba, D. Pavlidis, T. Karaduman,’First observation of bias oscillations in GaN Gunn diodes on GaN substrate", IEEE Trans. Electron Devices, Vol. ED-55, n6, pp.1563-1567, June 2008.

[7] M. Franz, J. B. Beyer, 'The travelling-wave IMPATT mode', IEEE Trans. Microwave Theory Tech., 26, 861, 1978.

[8] Y. Fukuoka, T. Itoh, 'Field analysis of a millimeter-wave GaAs double-drift IMPATT diode in the travelling-wave mode', IEEE Trans. Microwave Theory Tech, MTT-26, 861-865, November 1978.

[9] R. K. Mains, G. I. Haddad, 'Travelling-wave IMPATT amplifiers and oscillators', IEEE Trans. Microwave Theory Tech., MTT34(9), 965-971, March 1985.

[10] A. El Moussati, C. Dalle, "2D time-domain electromagnetic macroscopic numerical modelling on parallel computer: application to the mm-wave silicon DIMPATT diode", Journal of Computer Electronics, Vol. 7, pp. 34-42, 2008.

[11] B. Baraktaroglu, H. D. Shih, 'Millemeter-wave GaAs distributed IMPATT diodes', Trans. Electron Device Letters, EDL-4(11), 393-395, November 1983.

[12] C. Dalle, "2D time-domain numerical Maxwell/transport modelling for $\mathrm{THz}$ gallium nitride transferred electron device", International Journal of Numerical Modeling: electronic networks, devices and fields, Wiley, vol. 31, issue 2, March/April 2018

[13] R. O. Grondin, S. El-Ghazly, S. Goodnick, 'A review of global modeling of charge transport in semiconductors and full-wave electromagnetics', IEEE Trans. Microwave Theory Tech., MTT47(6), 817-829, 1999.

[14] C. Dalle, F. Dessene, J.-L. Thobel, "Theoretical investigation of THz GaN mesa Transferred-Electron Device by means of time domain energy/momentum modeling", IEEE Transactions on Electron Devices, Vol. 59, N¹2, pp. 3321-3326, December 2012.

[15] M. Lundstrom, 'Fundamentals of carrier transport', Second edition,Cambridge University Press, December 2000.

[16] K. Blotekjaer, 'Transport equations for electrons in two-valley semiconductors', IEEE Trans. Electron Devices, Vol. 17, pp. 38-47, 1970

[17] R. K. Mains, G. I. Haddad, P. A. Blakey, 'Simulation of GaAs IMPATT diodes including energy and velocity transport equations', IEEE Trans. Electron Devices, Vol. 30, n¹0, pp. 1327-1338, October 1983.

[18] A. de Mari,'An accurate numerical one-dimensional solution of the P-N junction under arbitrary transient conditons', Solid-State Electronics, SSE-11, 1021-1053, 1968.

[19] M. Shur, 'Influence of non-uniform field distribution on frequency limits of GaAs field effect transistors', Electronics Letters, 12, 615-616, 1976. 
[20] K. S. Yee, 'Numerical solution of initial boundary value problems involving Maxwell's equations in isotropic media', IEEE Trans. Antennas Propagat., AP-14(3), 302-307, may 1966.

[21] E. H. Twizell, "Computational methods for partial differential equations", Ellis Horwood Series Mathematics and its applications, John Wiley \& Sons, 1984.

[22] T. Namiki, 3-D-ADI-FDTD method - Unconditionally stable time-domain algorithm for solving full vector Maxwell's equations, IEEE Trans. Microwave Theory and Techniques, 48, $10,1743(2000)$.

[23] Cholewski. Mathematical methods for digital computers. Ed. E. L. Wachpress, Wiley, New-York (1967).

[24] M.-R. Friscourt, P. A. Rolland, A. Cappy, E. Constant, G. Salmer, 'Theoretical Contribution to the Design of Millimeter-Wave TEO's', IEEE Trans. Electron Devices, vol. ED-30, no. 3, pp. 223229, March 1983.

[25] U. S. Inan, R. A. Marschall, "Numerical Electromagnetics: The FDTD method", Cambridge University Press, 2011.

[26] G. Mur, "Absorbing boundary conditions for the finitedifference approximation of the time-domain electromagneticsfield equations", IEEE Trans. on Electronmagnetic compatibility, Vol. EMC-23, N², November 1981. 